\title{
Primary Care for Patients Infected with Human Immunodeficiency Virus
}

\author{
A Randomized Controlled Trial
}

\author{
Sheri A. Keitz, MD, PhD, Tamara L. Box, BS, Rick K. Homan, PhD, John A. Bartlett, MD,
} Eugene Z. Oddone, MD, MHSC

\begin{abstract}
OBJECTIVE: To measure the impact of a teaching intervention and to compare process and outcomes of care for HIV-infected patients randomly assigned to a general medicine clinic (GMC) or an infectious disease clinic (IDC) for primary care.
\end{abstract}

DESIGN: Prospective, randomized, controlled trial.

SETTING: University hospital in Durham, NC.

PATIENTS: Two hundred fourteen consecutive HIV-infected patients presenting for primary care.

INTERVENTION: Physicians at the GMC received HIV-related training and evidence-based practice guidelines.

MEASUREMENTS: Utilization of services, health-related quality of life, preventive and screening measures, and antiretroviral use for one year.

RESULTS: At baseline GMC patients were more likely to be African American (85\% vs $71 \%$; $P=.03$ ) and had lower baseline CD4+ cell counts than IDC patients $(262 \pm 269$ vs $329 \pm 275$; $P=.05)$. A similar and high proportion of patients in both groups received appropriate preventive care services including Pneumocystis carinii pneumonia (PCP) prophylaxis, pneumococcal vaccination, and antiretroviral therapy. Screening for TB was more frequent in GMC (89\% vs $68 \%$; $P=.001)$. In the year following randomization, GMC patients made more visits to the emergency department than IDC patients $(1.6 \pm 3.0$ vs $0.7 \pm 1.5 ; P=.05)$. Hospital use was higher for GMC patients with average length of stay $7.8 \pm 6.3$ days compared to $5.7 \pm 3.8$ days for IDC patients $(P=.01)$. In analyses, which adjust for potential baseline imbalances, these differences remained.

CONCLUSIONS: Targeted education in GMC achieved similar provision of primary care for GMC patients, yet use of health

Received from the Center for Health Services Research in Primary Care, Durham VA Medical Center, Durham, NC; and the Division of General Internal Medicine (SAK, TLB, RKH, EZO) and the Division of Infectious Diseases (JAB), Duke University Medical Center, Durham, NC.

This work was presented at the National Meeting of the Society of General Internal Medicine (April 1999, San Francisco).

Address correspondence and reprint requests to Dr. Keitz: VA Medical Center (14A), 508 Fulton St., Durham, NC 27705 (e-mail: sheri.keitz@duke.edu). care services was higher for this group. The delivery of adequate primary care is necessary but not sufficient to produce changes in health care utilization.

KEY WORDS: HIV infections; physician practice patterns; health services accessibility; continuity of patient care; ambulatory care; primary health care.

J GEN INTERN MED 2001;16:573-582.

$\mathbf{T}$ here is increasing pressure to treat human immunodeficiency virus (HIV) infection and acquired immune deficiency (AIDS) in the ambulatory setting with a dual purpose of providing high-quality care and lowering costs. There is, however, discussion over whether generalist or specialist physicians should provide this care. Prior studies have suggested that clinical experience in the care of the HIV-infected patient may predict patient outcomes. In the ambulatory setting, AIDS patients cared for predominantly by generalists had greater odds of hospitalization ${ }^{1}$ and greater lag time before receiving zidovudine ${ }^{2}$ when compared with patients cared for by specialist physicians. In addition, improved patient survival was observed in patients cared for by primary care physicians with greater experience in HIV care. ${ }^{3}$ With nonrandom allocation of patients in these studies, however, it is difficult to separate the provider effect from patient confounders.

There is additional controversy concerning which educational models are most effective at improving HIV care in the ambulatory setting. Internal medicine training directors and trainees report deficiencies in education relating to the ambulatory care of HIV-infected patients. ${ }^{4-6}$ In addition, surveys of physicians in training suggested that heavy loads of inpatient HIV care predicted negative attitudes and unwillingness to care for these patients in future practice, while experience in caring for HIVinfected patients in the ambulatory setting predicted positive attitudes. ${ }^{5}$

We conducted a randomized, controlled trial comparing process and outcome of care for HIV-infected patients delivered by generalists in a general medicine clinic (GMC) to the care delivered by specialists in an infectious disease clinic (IDC). To enhance the education necessary for the 
primary care of HIV-infected patients, we implemented a primary care educational program for the physicians in the GMC. Our primary goal was to determine if differences occurred with respect to hospitalization rates and lengths of stay, health related quality of life (HRQL) scores, and the delivery of appropriate preventive health care. We also evaluated the impact of the educational intervention on the provision of evidence-based services for HIV-infected patients. Both GMC and IDC incorporate trainees into the clinic process with the goal of providing hands-on training in the ambulatory care of HIV-infected patients. A second study objective was to examine each teaching model; thus, we also measured the proportion of care provided by trainees in each setting.

\section{METHODS}

\section{Clinic Sites}

We conducted a single site, randomized prospective trial of HIV-infected patients presenting to Duke University Medical Center (DUMC) for primary care. The 2 sites for patient assignment were: 1) the GMC, in which internal medicine residents, supervised by noninfectious diseases faculty attending physicians, provided care, and 2) the IDC, in which residents, infectious diseases (ID) fellows, and ID attending physicians provided care. At the outset of the study, the goal was to measure the number of study patients cared for by residents and fellows with faculty supervision.

During the study period, GMC provided care for approximately 5,000 general medicine patients. Less than 50 of these patients outside the study cohort were HIVinfected. Resident physicians saw patients in clinic onehalf day each week and were supervised by the same attending physician throughout the year. Two generalist faculty preceptors were available in clinic for 8 trainees each clinic half day. In IDC, residents, ID fellows, and ID attending physicians provided care for 1,100 HIV-infected patients. Residents and fellows were paired with one faculty preceptor who saw their own patients in addition to precepting the trainees. The study was approved and reviewed annually by the Institutional Review Board of DUMC.

\section{Inclusion/Exclusion Criteria}

Patient Eligibility. Eligible patients had a self-reported history of HIV infection and presented to DUMC for HIVrelated ambulatory care. Patients were eligible if they were uninsured, self-pay, or qualified for Medicaid or Medicare.

Because of constraints in billing structures for those who had private insurance, patients were excluded if they were privately insured $(n=188)$. They were also excluded if they were not competent to give informed consent as determined by the judgement of the attending physician available at the time of randomization $(n=2)$ or were residents of a nursing home $(n=2)$.

\section{Study Design}

Patient Enrollment. Enrollment occurred during the intake interview following the collection of baseline information by one of the study coordinators. The enrollment of patients was continuous beginning July 1994 and ending February 1997, when the target sample size was achieved. Patients were contacted at 3 months and 12 months following the date of randomization to assess vital status, HRQL, and health care utilization.

Informed Consent and Randomization. Written informed consent was obtained during intake interview. Randomization was stratified for risk group classification based on the patients' self-reported use of IV drugs versus no history of IV drug use and blocked in groups of 4 . The randomization sequence was maintained at a site separate from patient enrollment. Once the clinical site was known, the study coordinator assigned a physician to the patient and arranged the initial visit.

\section{Primary and Secondary Outcomes}

The primary outcome variables were hospitalization rate, length of stay, and proportion of patients hospitalized for 12 months following randomization. We also measured visits to ambulatory clinics and the emergency department. Outcomes involving hospitalizations and visits were obtained from the DUMC billing records and hospital information system. Hospital admissions and the number of hospital bed-days were tallied. Patient visits were recorded for all scheduled and unscheduled visits - both emergency department and acute care visits. We also obtained non-DUMC health care use through systematic queries of non-DUMC providers and hospitals. For hospitalizations at DUMC, we recorded primary discharge diagnosis from the dictated discharge summary for that hospital admission. When a dictated summary was not available, we recorded the stated discharge diagnosis from the hospital billing system by ICD-9 code. For outside hospitalizations, we did not have uniform access to information regarding discharge diagnosis.

Four a priori-defined process of care measures the provision of appropriate prophylaxis against PCP, tuberculosis screening, administration of pneumococcal vaccine, and documented discussions regarding advance directives) were assessed from medical record review. We also measured utilization of CD4+ cell counts and prescription of antiretroviral medications. Health-related quality of life (HRQL) was assessed with a HIV-specific survey. ${ }^{7,8}$

For prophylaxis against PCP, we documented care that complied with the U.S. Public Health Service recommendations. ${ }^{9-12}$ If patients were candidates for PCP prophylaxis, care was considered appropriate if they received trimethaprim-sulfamethoxazole, pentamidine, or dapsone. If patients were not candidates for PCP prophylaxis, care was considered appropriate only if they did not receive prophylaxis. Furthermore, we tracked CD4+ 
cell counts and changes in clinical status during the study period to document changes in eligibility for PCP prophylaxis.

We recorded documentation of tuberculosis screening and pneumococcal vaccination that met national recommendations. ${ }^{9-12}$ For the measurement of advance directives, we recorded evidence of a discussion of patient preferences for end-of-life care or formal do-not-resuscitate orders. Additionally, we recorded specific antiretroviral medications prescribed by physicians and changes in prescribed regimens.

\section{Physician Education}

We implemented an educational program that was mandatory for house staff and available for attending physicians in GMC. All primary care residents and an additional group of first and second year residents who expressed interest in HIV-related ambulatory care participated ( $n=49$ house officers). Teaching topics covered a curriculum intended to provide the foundation for appropriate primary care of HIV-infected patients (Table 1). In addition, quarterly case conferences were held to discuss specific cases and highlight the medical literature on the most current standards for HIV-related primary care. The initial study design included a parallel system in which study patients would be assigned primarily to a trainee with faculty supervision regardless of whether the trainees

\section{Table 1. Educational Program in General Medicine Clinic}

Didactic Series for Generalist Residents

Lecture series for study residents; 8-12 lectures each year of patient enrollment.

Lecture topics included:

- Orientation and overview of practice guidelines

- H and P, labs - the first visit and onward

- PCP and other prophylaxis

- Antiretroviral therapy - to have or have not?

- Opportunistic infections - what to watch out for

- Women and HIV - special things to consider

- Doctoring the dying patient

- HIV from the outside - the dermatology of HIV

- HIV-related protocols - keeping informed

- Primary prevention: halting the spread of HIV

Clinical Practice Guidelines

- Local clinical practice guidelines were developed and distributed (see Appendix). Guidelines were based on the medical literature, including the Agency for Health Care Policy and Research Clinical Practice guidelines for evaluation and management of early HIV infection and the USPHS/IDSA guidelines for the prevention of opportunistic infections in persons infected with human immunodeficiency virus. Supporting documents were discussed and distributed.

Case Conferences for Generalist and Specialist Physicians

- Conferences used a case-based format to address relevant new issues in the primary care of HIV-infected patients. These workshops were jointly run by 1 generalist and 1 specialist. Four case conferences were held for each year of enrollment. were in GMC or IDC. In GMC the trainees were all residents whereas in IDC the trainees were both residents and fellows.

\section{Statistical Analysis}

We conducted an intention-to-treat analysis. All patients were followed for 12 months postenrollment, until death or lost to follow up. Wilcoxon rank sums or $\chi^{2}$ tests were used to assess baseline differences between groups and to perform primary outcome comparisons. Because of the baseline differences between the groups in 2 variables, we supplemented the primary analysis with a mixed model analysis or logistic regression as appropriate. In these models, race, CD4 count, distance from Duke, IV drug use, and treatment group were independent variables, and hospitalization rate, utilization of the emergency department, and length of hospital stay were dependent variables. A $P$ value of $\leq .05$ was considered significant.

The study was designed to exclude clinically important differences between GMC and IDC in all 3 of the primary outcome variables. The variable most limiting sample size was hospitalization rate. Using a projected hospitalization rate $(0.84 \pm 0.84$ hospitalizations per patient per year) based on the literature and local pilot information, we defined a clinically meaningful difference between groups of $30 \%$. This required a total of 200 patients (one-sided $\alpha=0.05$, power $=80 \%$ ), allowing for a $10 \%$ drop out.

\section{RESULTS}

\section{Patients}

From July 1994 through February 1997 we approached 238 consecutive patients who met inclusion criteria. We randomized and followed 214 of these patients (90\%; Fig. 1). Nineteen patients died during the study period (9\%) and 15 were lost to follow up (7\%; Fig. 1).

\section{Baseline Patient Characteristics}

Patients randomized to GMC $(n=105)$ and IDC $(n=109)$ were similar with respect to baseline demographic variables except for race and CD4 count (Table 2). Patients randomized to GMC had lower CD4 counts at baseline (262 vs 329; $P=.05)$ and were more likely to be African American (85\% vs $71 \% ; P=.03)$.

\section{Physician Level of Training and Number of Patients Followed}

In GMC all patients were randomly assigned to internal medicine residents supervised by non-ID attending physicians ( $n=49$ resident physicians). Thus, residents in their first through third year of postgraduate training followed $100 \%$ of patients. Patients randomized to IDC were assigned either to internal medicine residents $(n=10)$ with faculty supervision, ID fellows $(n=4)$ with faculty supervision, or ID faculty members $(n=4)$. Residents followed 


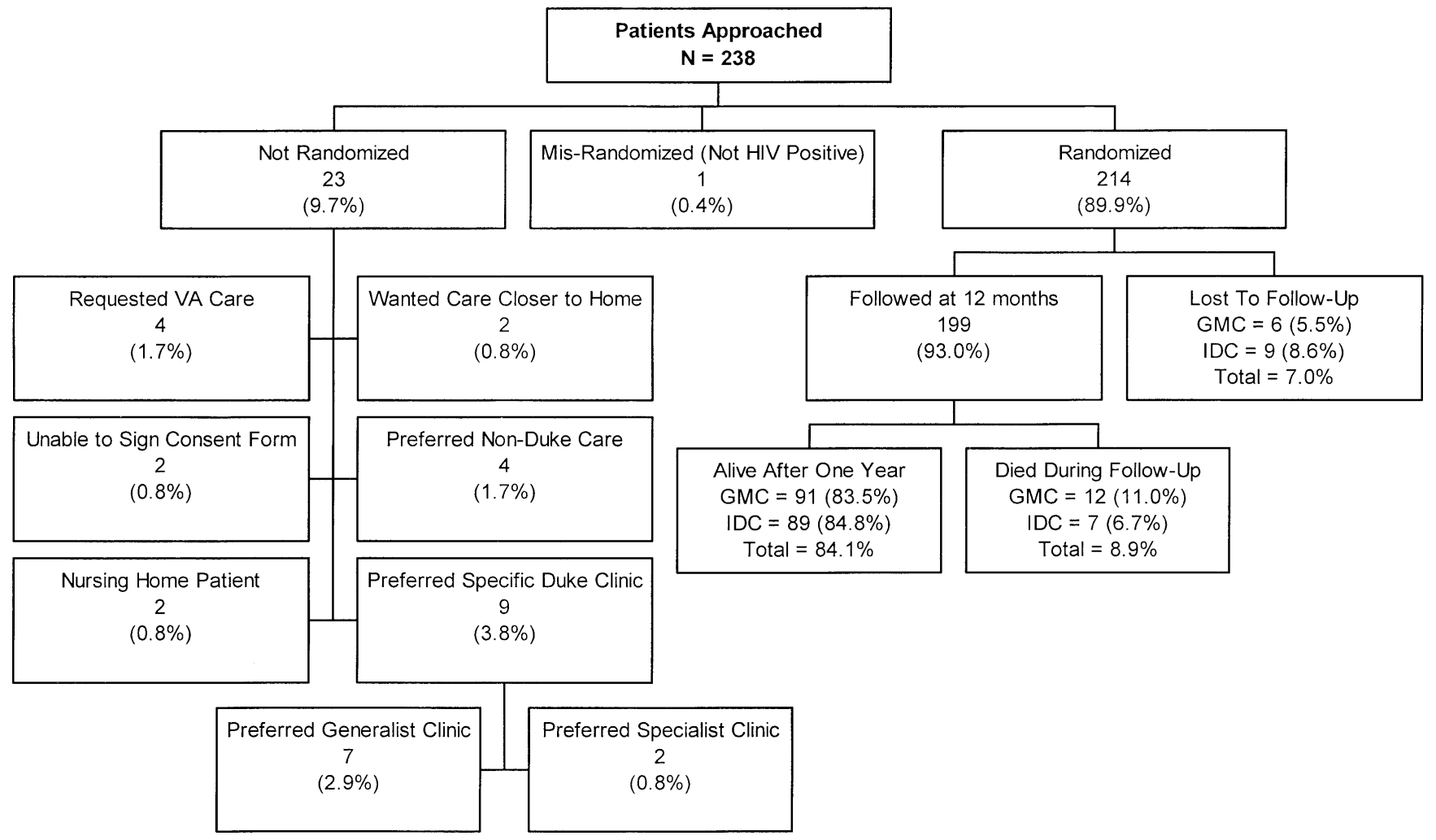

FIGURE 1. Randomization and patient follow-up.

$35 \%$ of patients, fellows followed $41 \%$ of patients, and attending physicians followed $24 \%$.

\section{Number of Patients Followed per Physician}

The average number of patients followed by each physician in GMC was $2.8 \pm 1.7$. IDC Physicians from IDC followed, on average $10.8 \pm 6.2$ patients. When assessed as number of study patients followed by individual physicians (Fig. 2), the majority of residents in GMC (65\%) cared for 2 to 5 patients and only $6 \%$ cared for greater than 5 . In contrast, $40 \%$ of the residents in IDC cared for greater than 5 patients and the all of the fellows in IDC cared for greater than 5 .

\section{Outpatient Visits}

Table 3 summarizes annual visit rates (number of visits per patient per year). There was no difference in mean visit rate to the clinic to which the patient was randomized over the 1-year study period. Patients randomized to GMC, however, were more likely to "cross over" and visit the IDC compared to patients randomized to IDC who visited the GMC $(0.56 \pm 1.57$ crossover visits per patient per year for GMC vs $0.11 \pm 0.62$ crossover visits per patient per year for IDC; $P=.01$ ).

Patients in the 2 study groups had similar access to the clinic to which they were randomly assigned. The average number of days to first scheduled appointment was similar between groups $(22.4 \pm 36.8$ days for GMC vs $26.1 \pm 24.3$ days for IDC). A majority of patients kept at least 1 appointment with the physician to whom they were assigned in both GMC and IDC (77.9\% vs 67.6\%, $P=.09$ ). A larger majority of study patients kept at least 1 appointment to see any provider in the clinic to which they were assigned (86.2\% for GMC vs $81.9 \%$ for IDC). More than half of the patients assigned to each group received greater than $50 \%$ of their total ambulatory care from the clinic to which they were assigned (53.2\% for GMC vs $58.1 \%$ for IDC). Also patients in the 2 study groups had similar attendance in the GMC and IDC. Of the 1,330 total ambulatory care visits scheduled by patients randomized to GMC and the 1,130 total ambulatory care visits scheduled by patients randomized to IDC, approximately two thirds of visits were kept (68.1\% for GMC and 68.9\% for IDC).

The mean visit rates for medical subspecialty clinics, surgical clinics, and ob-gyn were also similar between groups. However, GMC patients made a greater number of visits to the emergency department than did IDC patients $(1.6 \pm 3.0$ visits per patient per year for GMC vs $0.7 \pm 1.5$ visits per patient per year for IDC; $P=.05$ ). Six percent of the emergency department utilization occurred outside of DUMC.

\section{Hospital Use}

In total, 62 patients were hospitalized 123 times during the study period (Table 4). Patients randomly 
Table 2. Baseline Characteristics of Patients According to Study Group

\begin{tabular}{|c|c|c|c|}
\hline Characteristic & $\begin{array}{l}\text { Generalist } \\
(N=109)\end{array}$ & $\begin{array}{l}\text { Specialist } \\
(N=105)\end{array}$ & $\begin{array}{c}P \\
\text { Value* }\end{array}$ \\
\hline Age, y & $35.0 \pm 8.2$ & $34.3 \pm 9.0$ & .35 \\
\hline Male gender, \% & 58.7 & 63.8 & .45 \\
\hline Race, \% & & & .03 \\
\hline White, non-Hispanic & 11.9 & 20.0 & \\
\hline $\begin{array}{l}\text { African American, } \\
\text { non-Hispanic }\end{array}$ & 85.3 & 71.4 & \\
\hline Other & 2.8 & 8.6 & \\
\hline Education, \% high & & & \\
\hline school graduate & 53.2 & 59.1 & .39 \\
\hline Marital status, \% & & & .91 \\
\hline Single & 65.1 & 66.7 & \\
\hline Married & 7.3 & 8.6 & \\
\hline Separated & 12.8 & 13.3 & \\
\hline Other & 14.8 & 11.4 & \\
\hline Children, \% with & 55.1 & 57.1 & .76 \\
\hline Employment, \% & & & .99 \\
\hline Employed full time & 12.8 & 12.4 & \\
\hline Employed part time & 2.8 & 3.8 & \\
\hline Unemployed & 34.0 & 31.4 & \\
\hline Disabled & 37.6 & 39.1 & \\
\hline Other & 12.8 & 13.3 & \\
\hline \multicolumn{4}{|l|}{ Distance to Duke } \\
\hline \multicolumn{4}{|l|}{ University Medical } \\
\hline Center, miles & $55.2 \pm 49.1$ & $69.1 \pm 61.6$ & .25 \\
\hline CD $4+$ count, cells $/ \mu \mathrm{L}$ & $262.0 \pm 268.5$ & $329.2 \pm 275.3$ & .05 \\
\hline $\begin{array}{l}\text { Ever used IV drugs, } \\
\% \text { yes }\end{array}$ & 31.2 & 32.4 & .85 \\
\hline How HIV disease & & & \\
\hline was contracted, \% & & & .63 \\
\hline Heterosexual & 44.9 & 35.2 & \\
\hline Men who have sex & & & \\
\hline with men & 16.5 & 22.9 & \\
\hline IV drug use & 15.6 & 17.1 & \\
\hline $\begin{array}{l}\text { IV drug use and } \\
\text { additional risk }\end{array}$ & & & \\
\hline factor & 19.3 & 20.0 & \\
\hline Other & 3.7 & 4.8 & \\
\hline Health insurance, \% & & & .08 \\
\hline Medicaid & 34.9 & 23.8 & \\
\hline Other & 65.1 & 76.2 & \\
\hline
\end{tabular}

* $\mathrm{P}$ values are based on Wilcoxon rank sum tests or $\chi^{2}$ tests, as appropriate.

assigned to GMC experienced annual admission rate of $0.8 \pm 1.7$ compared to $0.4 \pm 0.8$ for patients assigned to IDC $(P=.11)$. For those patients who were hospitalized, there was approximately a $50 \%$ greater average length of stay for GMC patients $(9 \pm 7$ days vs $5.3 \pm 3.7$ days; $P=.01)$. Seven percent of hospitalizations occurred outside of DUMC. Discharge diagnoses relating to the hospitalizations are summarized in Table 5 . We were able to identify primary discharge for the 114 hospitalizations that were in the DUMC system (93\% of total admissions). Nearly half of these admissions (49/114) were related to AIDS defining or HIV-related conditions. The majority of these 49 admissions were in the GMC group (40/49). Twenty-five admissions were for medical comorbidities not directly related to HIV infection such as diabetes mellitus, peritonitis, cholangitis, seizures, and stroke. The majority of these admissions were in the GMC group as well $(19 / 25)$. We did not have discharge diagnoses for the outside hospitalizations.

In adjusted analyses that accounted for imbalances in baseline variables, differences between clinics remained for number of hospital days $(P=.05)$ and utilization of the emergency department $(P<.05)$. There was a trend for greater hospitalization rate in patients randomized to GMC $(P=.08)$.

\section{Process of Care}

Process of care measures are based on medical record review for 198 of 214 patients (93\%). Using nationally accepted criteria for PCP prophylaxis, ${ }^{9-12}$ appropriate PCP prophylaxis was provided for $93 \%$ of patients in GMC and $98 \%$ of patients in IDC $(P=.18)$. Appropriate TB screening was documented $89 \%$ of the time in GMC and $68 \%$ of patients in $\operatorname{IDC}(P=.001)$. There was evidence of pneumococcal vaccination for $68 \%$ of the patients from GMC and 59\% of the patients from IDC $(P=.16)$. We found evidence of discussion concerning advance directives in $31 \%$ of medical records of GMC patients and $21 \%$ percent of medical records of patients randomized to IDC $(P=.11)$. Of patients eligible for prophylaxis against Mycobacterium avium complex, 66\% of GMC patients and 56\% of IDC patients received prophylaxis. Seven patients in GMC and 10 patients in IDC participated in other clinical trials regarding care for HIV-infected patients.

Overall use of antiretroviral drugs was similar in GMC as compared with IDC. Similar numbers of patients received no antiretroviral medication at any time during the study period (20\% for GMC vs $24 \%$ in IDC), 1 antiretroviral (47\% for GMC vs $41 \%$ for IDC), a maximum of 2 antiretrovirals ( $20 \%$ for GMC vs $21 \%$ for IDC), or a maximum of 3 antiretrovirals during the study period (13\% for GMC vs $14 \%$ for IDC). In patients whose CD4 count was $<200$ lymphocytes $/ \mu \mathrm{L}$ at any time in the study, $91 \%$ received at least 1 antiretroviral. The average number of antiretrovirals prescribed for those patients who received antiretrovirals was $1.33 \pm 0.54$ for patients followed by IDC physicians and $1.32 \pm 0.52$ for patients followed by GMC physicians. Figure 3 shows antiretroviral use by clinic and study year.

\section{Health-related Quality-of-life Scores}

HIV-specific HRQL scores are presented in Table 6. There were no statistically significant differences between GMC and IDC patients in HRQL summary scores for physical health or mental health at baseline or at 12 months. In all patients, however, there was decline in physical health summary scores and mental health summary scores from baseline to 12 months. The decrease in summary scores was significant in GMC for physical health summary $(P=.0002)$ and for mental health summary $(P=.0002)$. The decrease in physical health 


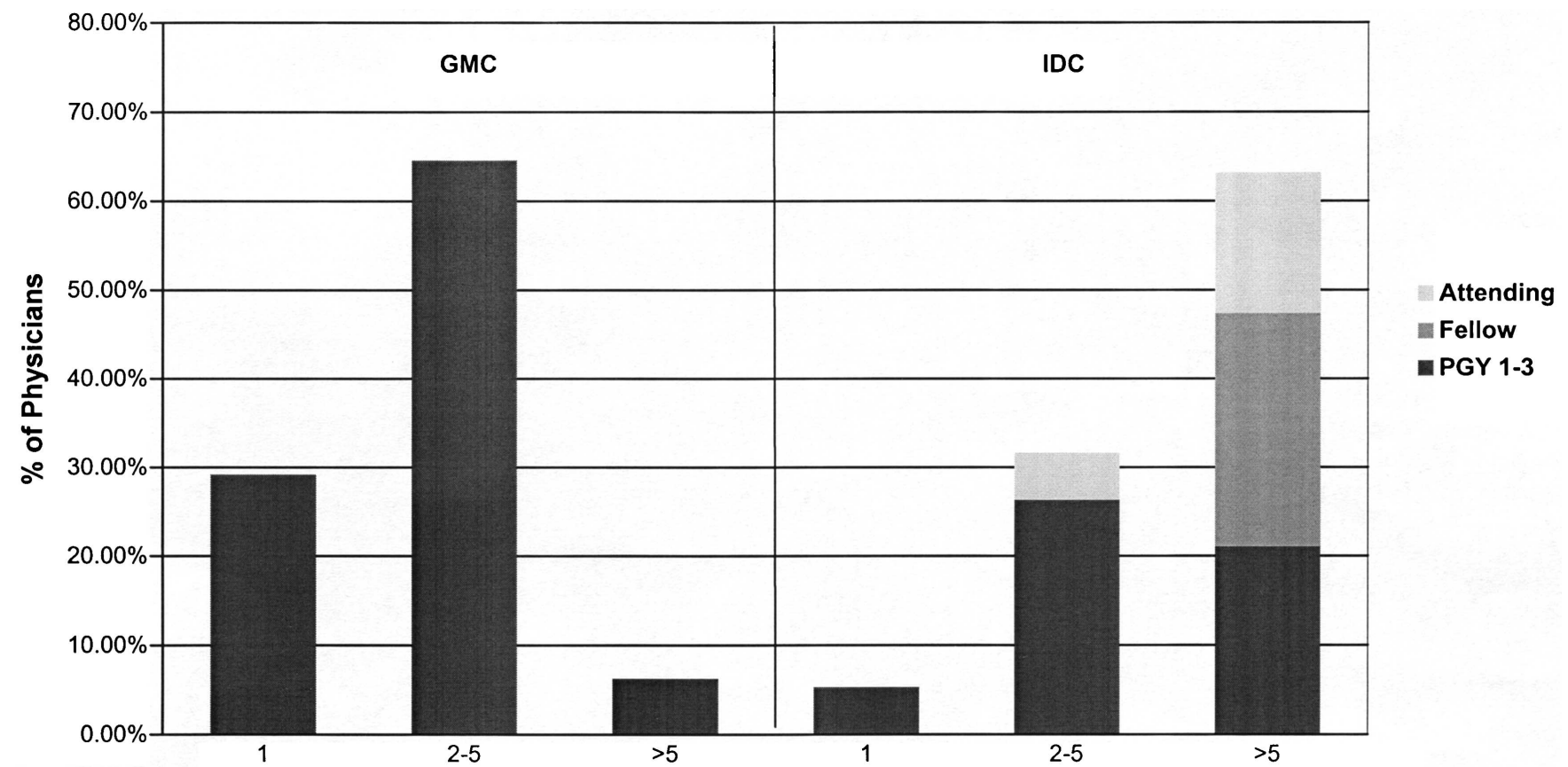

Number of Patients Treated During Study

FIGURE 2. Number of patients followed per physician during the study period. Bars show the percentage of physicians who followed 1 study patient, 2-5 study patients, or $>5$ study patients. The color indicates level of training. The first 3 bars represent within-study experience of the physicians in GMC and the final 3 bars represent within-study experience of the physicians in IDC.

summary scores was also significant in IDC $(P=.0014)$ with a trend towards significance in mental health summary scores $(P=.08)$.

\section{DISCUSSION}

We examined process and outcomes of care for HIVinfected patients randomly assigned to 2 different teaching settings, IDC and GMC. Despite remarkably similar process of care, potentially due to a teaching program for residents in GMC, we found a difference in health care utilization between patients randomly assigned to these clinics. Specifically, utilization of the emergency department and the number of hospital days in the 12-month follow-up period were significantly higher for patients randomized to GMC. These differences remained even after adjusting for baseline imbalances between the 2 patient groups.

Increased hospital use for patients cared for by generalist physicians has been previously suggested in a

Table 3. Outpatient Visits

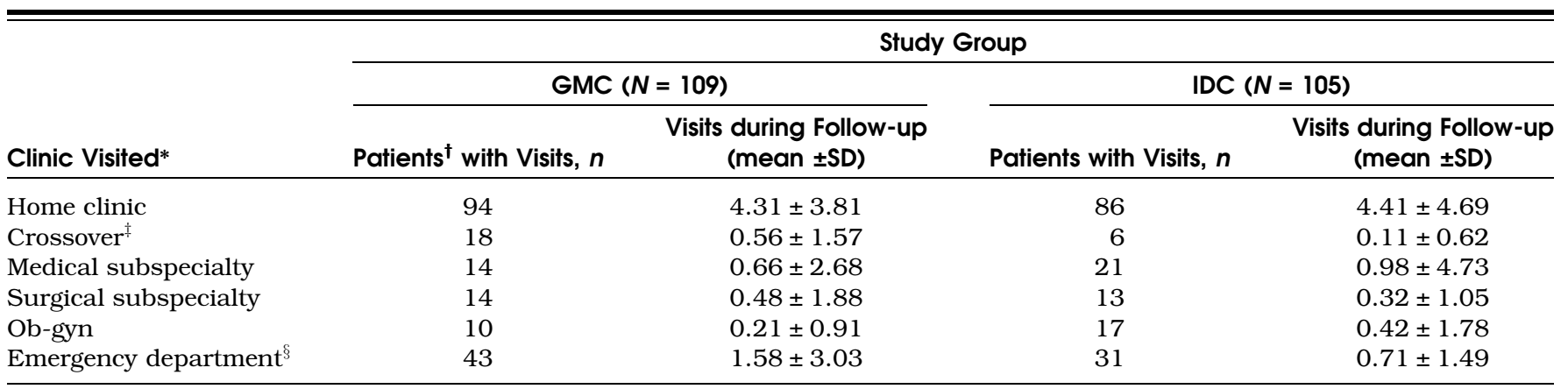

* Home clinic refers to the clinic to which the patient was randomized. Crossover refers to visits to the clinic to which the patient was not randomized.

${ }^{\dagger}$ The total number of patients shown in the clinic categories exceeds the total number of patients in the study (214) because some patients had visits to more than one clinic category.

${ }^{\ddagger} \mathrm{P}=.05$.

${ }^{\S} \mathrm{P}=.01 ; \mathrm{P}$ values compare mean visits during follow-up. 
Table 4. Hospital Use

\begin{tabular}{lccc}
\hline \hline & $\begin{array}{c}\text { GMC } \\
(N=109)\end{array}$ & $\begin{array}{c}\text { IDC } \\
(N=105)\end{array}$ & $\begin{array}{c}\text { Unadjusted } \\
\boldsymbol{P} \text { Value }\end{array}$ \\
\hline Proportion admitted & $33 \%$ & $25 \%$ & .18 \\
Annual admission rate & $0.8 \pm 1.7$ & $0.4 \pm 0.8$ & .11 \\
Average length of stay* & $9.0 \pm 7.0$ & $5.3 \pm 3.7$ & .01 \\
\hline
\end{tabular}

* Calculation based upon hospitalized patients only.

retrospective evaluation of patients enrolled in the New York State Medicaid program. ${ }^{1}$ Patients cared for by generalists had greater odds of being hospitalized (odds ratio 2.2) and, if hospitalized, had a longer length of stay. Our results prospectively confirm that utilization of hospital services was higher for the patients followed by GMC physicians. Furthermore, our study extends previous efforts in that we defined and measured key components of high quality primary care for HIV-infected patients. For the most part, the provision of these components of care was excellent (e.g., over 90\% of patients received appropriate PCP prophylaxis). In fact, the only differences in process of care (tuberculosis screening) favored the care for GMC patients.

Our findings contrast the previous work of Kitahata et al. ${ }^{3}$ who performed a retrospective medical record review evaluating AIDS patients cared for by family physicians within a staff model-HMO. Kitahata et al. reported that more experienced doctors (all generalists) were more likely to prescribe appropriate PCP prophylaxis, whereas in our cohort, provision of PCP prophylaxis was high and comparable between GMC and IDC. The physicians in the Kitahata study, however, had received no targeted education in HIV-care. Kitahata et al.'s study also documented that patient survival was associated with the experience of individual providers. The experience garnered from the care of even small numbers of HIV infected patients (2 to 5) led to improved patient outcomes, specifically a lower risk of death. While our study was not powered to detect differences in mortality between the patient groups, the numbers of patients followed per physician in Kitahata et al. is consistent with the numbers of patients cared for by most of the providers in our study.

Use of antiretroviral agents is a big determinant of survival and their use is associated with lower use of hospital services. ${ }^{13-15}$ Previous studies have linked physician experience to utilization of antiretroviral medications. ${ }^{2,3}$ Markson et al. ${ }^{2}$ performed a retrospective analysis of New York State Medicaid claims comparing utilization of zidovudine therapy by generalist versus specialist physicians. In our prospective cohort, however, the overall percentage of patients who were prescribed antiretroviral medications and the number of medications prescribed was similar, even when controlling for temporal trends.

In our study, differences in utilization may be related to differences in overall and HIV-specific clinical experience between the 2 groups of physicians. In IDC, of the 18 providers who cared for patients, only $56 \%$ of them were residents, the remaining physicians being fellows and attending physicians. In GMC, all of the 49 doctors were residents supervised by non-ID attending physicians. Residents and attending physicians might be quite different in their use of strategies such as telephone medicine or reliance on midlevel providers to handle acute issues between visits.

Not only did the doctors differ in level of training, they differed in the amount of experience that was provided

Table 5. Description of Hospitalizations

\begin{tabular}{|c|c|c|c|c|}
\hline \multirow[b]{2}{*}{ Primary Discharge Diagnosis } & \multicolumn{4}{|c|}{ Study Group } \\
\hline & $\begin{array}{c}\text { Patients with } \\
\text { Hospitalizations, }{ }^{\ddagger} n \\
(N=36 \text { Patients })\end{array}$ & $\begin{array}{l}\text { Hospitalizations, } n \\
\text { ( } N=84 \text { Admissions) }\end{array}$ & $\begin{array}{c}\text { Patients with } \\
\text { Hospitalizations, }{ }^{\ddagger} n \\
(N=26 \text { Patients })\end{array}$ & $\begin{array}{c}\text { Hospitalizations, } n \\
\text { ( } N=39 \text { Admissions) }\end{array}$ \\
\hline AIDS defining condition & 16 & $22^{*}$ & 2 & $2^{*}$ \\
\hline HIV-related condition & 12 & $18^{\dagger}$ & 6 & $7^{\dagger}$ \\
\hline Substance abuse and psychiatry & 6 & 11 & 2 & 3 \\
\hline Ob-gyn & 2 & 2 & 4 & 4 \\
\hline Other medical hospitalizations & 10 & 19 & 5 & 6 \\
\hline Outside hospitalizations & 2 & 2 & 5 & 7 \\
\hline
\end{tabular}

* Includes diagnoses characterized as AIDS defining by the CDC surveillance definition. GMC discharge diagnoses were Candida esophagitis $(\mathrm{n}=5)$, Cryptococcus ( $\mathrm{n}=2)$, Cryptosporidium $(\mathrm{n}=1)$, lymphoma $(\mathrm{n}=5)$, MAC $(\mathrm{n}=2), P C P(\mathrm{n}=4)$, toxoplasmosis $(\mathrm{n}=2)$, and tuberculosis $(\mathrm{n}=1)$. IDC diagnoses were lymphoma $(\mathrm{n}=1)$ and PCP $(\mathrm{n}=1)$.

${ }^{\dagger}$ Includes those diagnoses related to HIV infection but that were not AIDS defining. GMC discharge diagnoses were dementia ( $\mathrm{n}=1$ ), pneumonia $(\mathrm{n}=10)$, HIV esophageal ulcer $(\mathrm{n}=1)$, HIV myelopathy $(\mathrm{n}=1)$, HIV nephropathy $(\mathrm{n}=2)$, and HIV disease without other specific diagnosis $(\mathrm{n}=3)$. IDC diagnoses were esophageal ulcer $(\mathrm{n}=2)$, pneumonia $(\mathrm{n}=2)$, and HIV disease without other specific diagnosis $(\mathrm{n}=3)$. ¥ The total number of patients shown in the discharge diagnosis categories exceeds the total number of patients with hospitalizations because some patients had admissions to more than 1 category. 


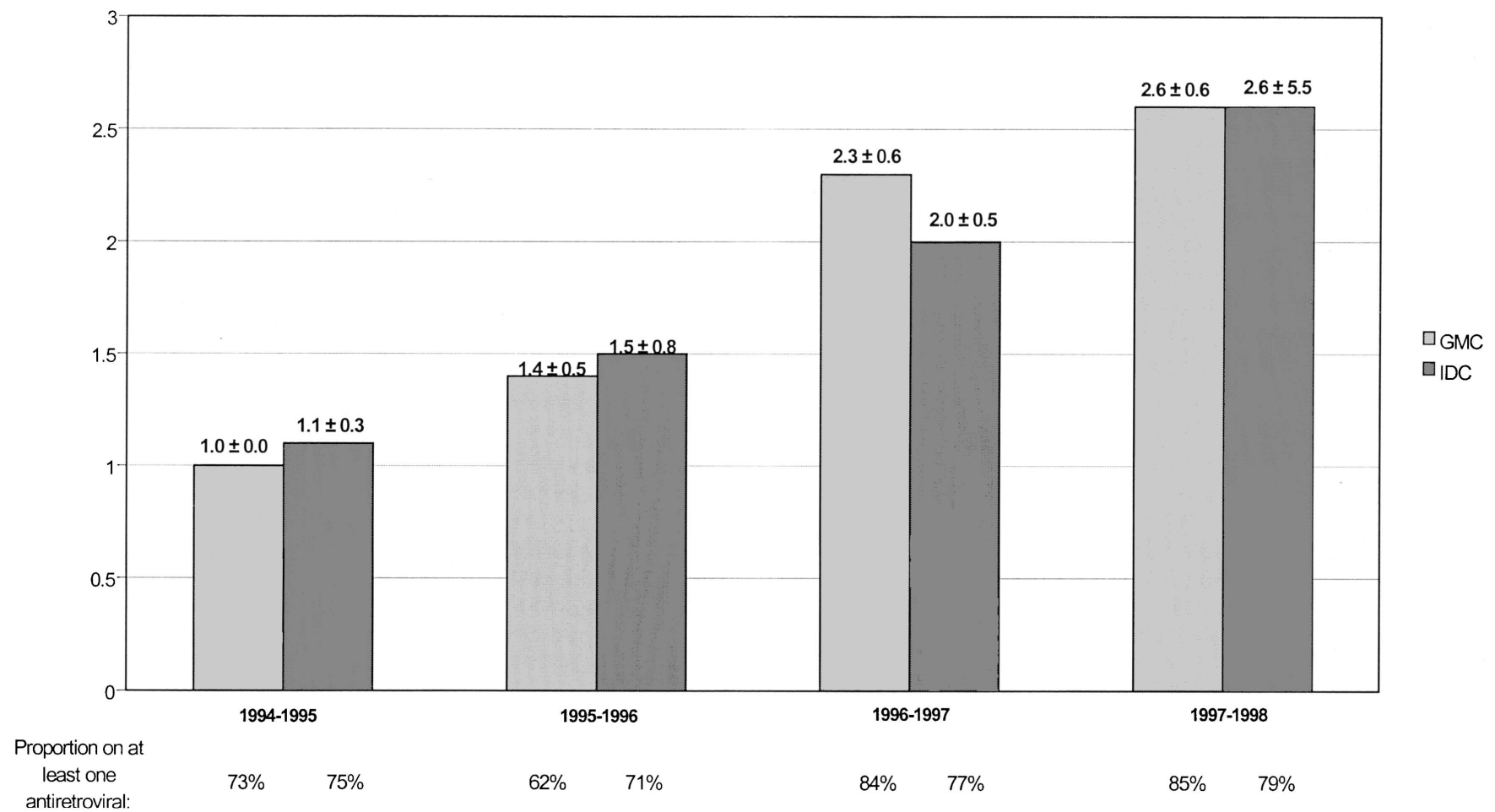

FIGURE 3. Antiretroviral use. The time periods represent 4 nonoverlapping time periods approximately representing the 4 years of the study. Because we completed follow-up in February of 1998, the fourth time period is less than 1 year. The average number of antiretrovirals is reported for any patient followed during that time; therefore, patients may be included in more than 1 time period. The proportion of patients on at least 1 antiretroviral during each of the study years is also reported.

within the study cohort. In GMC, medical residents with little or no prior HIV-related ambulatory care experience assumed primary responsibility for all of the HIV-infected patients that were randomly assigned to that clinic. Because there were many GMC physicians who provided care, each individual physician cared for few patients in the context of the study. In contrast, in IDC, fellows and attending physicians provided care for more HIV-infected patients both within the study. Although IDC physicians averaged $10 \mathrm{HIV}$-infected study patients, they also cared for a significant number of HIV-infected patients outside the study. In contrast, physicians in GMC, cared for few, if any, HIV-infected patients outside of the study cohort. Thus, physician experience was vastly different between the 2 groups of doctors.

In addition to differences between the physicians in the 2 groups, there were important differences in clinic structure and support. The ID attending physicians are available in clinic multiple half days each week and may accommodate acute needs for the patient population more readily than in the GMC. In contrast, residents in GMC had clinic one half day each week and they were not organized into practice groups or coverage teams. When their patients needed care between scheduled clinic visits, GMC residents may have been less available. Nursing and social work support were organized differently in the 2 different clinic settings. Staff in IDC had a very targeted focus on the case management of HIV-infected patients with 3 social workers available. In contrast, GMC is set up to manage the more diverse needs of a population with multiple chronic and acute diseases with only 1 social worker available.

The population we studied was exclusively uninsured or government insured and nearly $80 \%$ of patients were black. Our results in this population are consistent with results from the HIV Cost and Services Utilization Study, a national survey based on a sample of the adult U.S.

Table 6. Physical and Mental Health-Related Quality of Life Summary Scores*

\begin{tabular}{lcccc}
\hline \hline & \multicolumn{2}{c}{ Baseline } & \multicolumn{1}{c}{ 12 Month } \\
\cline { 2 - 4 } & IDC $(\boldsymbol{N}=\mathbf{1 0 2})$ & GMC $(\boldsymbol{N}=108)$ & & IDC $(\boldsymbol{N}=\mathbf{5 6})$ \\
\hline Physical health summary & $42.8 \pm 13.3$ & $42.1 \pm 12.2$ & $37.2 \pm 11.7$ & $35.7 \pm 11.1$ \\
Mental health summary & $40.2 \pm 12.7$ & $42.4 \pm 12.5$ & $37.2 \pm 11.7$ & $37.8 \pm 11.8$ \\
\hline
\end{tabular}

* Scores on the MOS-HIV HRQL questionnaire range from $O$ (lowest) to 100 (highest). 
population infected with HIV. ${ }^{16,17}$ In the HCSUS, the annual emergency department visit rate in the Medicaid population was $30 \%$ compared to $36 \%$ in this study. Additionally, the proportion of Medicaid patients hospitalized in 6 months was $25 \%$ compared to $29 \%$ over 1 year in this study.

One of our study objectives was to expose a large number of trainees to HIV-infected patients in the ambulatory setting. At the outset of the study, Duke residency training focused predominantly on inpatient care for the HIV-infected. Only a handful of residents cared for patients outside of the hospital. This is consistent with a nationwide survey of program directors in internal medicine that reported that the overwhelming majority of residents completed their training without ever caring for any HIVinfected patients in the ambulatory setting. ${ }^{4}$

By the conclusion of the study, 63 trainees had cared for ambulatory HIV-infected patients. Three quarters of these trainees provided care in GMC for all of the HIVinfected patients followed in GMC, whereas a small number of trainees in IDC provided care for $75 \%$ of the patients randomized to that group. Thus, we did expose a greater number of residents to direct patient care in GMC. Furthermore, when comparing measures of preventive and primary care, the residents in GMC provided remarkably good care, equal to that provided through the physicians with more experience in the IDC.

There are several important limitations to our study. Our study was performed in a single academic medical center in the context of a resident-run clinic. Another limitation relates to the ongoing rapid change in available treatment choices and standard of care. While we captured information on patterns of care during the transition to the era of highly active antiretroviral therapy (HAART), we do not have enough information to report on the usage of these agents. Despite these limitations, our study is the first in which care provided by generalist and specialist physicians for HIV-infected patients was compared in the setting of random allocation of patients to different primary care settings. We enrolled and randomized $89 \%$ of eligible patients who sought HIV-related primary care in our setting and had follow-up information on $93 \%$ of patients, which attests to high internal validity.

In summary, patients assigned to specialist clinic had significantly less use of hospital services than those patients assigned to generalist clinic. These differences are not explained by differences in the receipt of wellestablished components of evidence-based primary care such as PCP prophylaxis, screening for TB, and administration of pneumococcal vaccination. Even aspects of HIV care in which the standards were rapidly changing, such as antiretroviral use, were provided equally in the 2 groups. Some unmeasured aspect of physician experience in HIV care or structure of the clinics may account for these differences. Attaining the clinical goal of high quality care and limited use of expensive hospital services for HIV-infected patients may require a greater degree of subspecialty input or a restructuring of the support services of the clinic. The provision and retention of evidence-based interventions is not sufficient to achieve these two goals. In addition, the tension remains between the need to train physicians to become experienced clinicians in the care of HIV-infected patients and the need to reap the benefits of relying on already experienced clinicians to manage scarce resources in the current, costconscious health care environment.

We are indebted to Drs. John Hamilton and Charles Bennett for their thoughtful reading of this manuscript. In addition, this study could not have been performed without the dedication of the internal medicine house-staff in the Department of Medicine at Duke University Medical Center.

This work was supported by a grant from the Robert Wood Johnson Foundation and the Lawrence S. Linn Foundation for the study of quality of life in HIV-infected patients.

\section{REFERENCES}

1. Turner BJ, McKee L, Fanning T, Markson LE. AIDS specialist versus generalist ambulatory care for advanced HIV infection and impact on hospital use. Med Care. 1994;32:902-16.

2. Markson LE, Cosler LE, Turner BJ. Implications of generalists' slow adoption of zidovudine in clinical practice. Arch Intern Med. 1994;154:1497-504.

3. Kitahata MM, Koepsell TD, Deyo RA, Maxwell CL, Dodge WT, Wagner EH. Physicians' experience with the acquired immunodeficiency syndrome as a factor in patients' survival. N Engl J Med. 1996;334:701-6.

4. Hayward RA, Kravitz RL, Shapiro MF. Program directors' attitudes towards residents' care of patients who have AIDS. J Gen Intern Med. 1991;6:18-26.

5. Hayward RA, Weissfeld JL. Coming to terms with the era of AIDS: attitudes of physicians in U.S. residency programs. J Gen Intern Med. 1993;8:10-8.

6. Shapiro MF, Hayward RA, Guillemot D, Jayle D. Residents experiences in, and attitudes toward, the care of persons with AIDS in Canada, France, and the United States. JAMA. 1992;268:510-5.

7. Wu AW. A health status questionnaire using 30 items from the Medical Outcomes Study. Preliminary validation in persons with early HIV infection. Med Care. 1991;29:786-98.

8. Revicki DA, Sorensen S, Wu AW. Reliability and validity of physical and mental health summary scores from the Medical Outcomes Study HIV Health Survey. Med Care. 1998;36:126-37.

9. Anonymous. Recommendations for prophylaxis against Pneumo cystis carinii pneumonia for adults and adolescents infected with human immunodeficiency virus. Morb Mortal Wkly Rep. 1992;41(RR-4):1-11.

10. Anonymous. 1997 USPHS/IDSA guidelines for the prevention of opportunistic infections in persons infected with human immunodeficiency virus: disease-specific recommendations. USPHS/IDSA Prevention of Opportunistic Infections Working Group. Clin Infect Dis. 1997;25(Suppl 3):313-5.

11. Anonymous. USPHS/IDSA guidelines for the prevention of opportunistic infections in persons infected with human immunodeficiency virus: disease-specific recommendations. USPHS/IDSA Prevention of Opportunistic Infections Working Group. Clin Infect Dis. 1995;21(Suppl 1):32-43.

12. Anonymous. 1997 USPHS/IDSA guidelines for the prevention of opportunistic infections in persons infected with human immunodeficiency virus. Ann Intern Med. 1997;127:922-46. 
13. Gebo KA, Chaisson RE, Folkemer JG, Bartlett JG, Moore RD. Costs of HIV medical care in the era of highly active antiretroviral therapy. AIDS. 1999;13:963-9.

14. Mouton Y, Alfandari S, Valette M, et al. Impact of protease inhibitors on AIDS-defining events and hospitalizations in 10 French AIDS reference centres. Federation National des Centres de Lutte contre le SIDA. AIDS. 1997;11:F101-F105.

15. Torres RA, Barr M. Impact of combination therapy for HIV infection on inpatient census [letter]. N Engl J Med. 1997;336: 1531-2.

16. Bozzette SA, Berry SH, Duan N, et al. The care of HIV-infected adults in the United States. HIV Cost and Services Utilization Study Consortium. N Engl J Med. 1998;339:1897-904.

17. Shapiro MF, Morton SC, McCaffrey DF, et al. Variations in the care of HIV-infected adults in the United States: results from the HIV Cost and Services Utilization Study. JAMA. 1999;281:2305-15.

\section{APPENDIX}

\section{Local Clinical Practice Guidelines}

HIV practice guidelines covered the following topics:

- Staging of HIV infection

- Conditions included in the 1993 AIDS surveillance definition (MMWR December 1992; 41.)

- Labs and ongoing evaluation: the first visit

- PCP prophylaxis

- Starting antiretroviral therapy

- Ongoing antiretroviral therapy

- Mycobacterium tuberculosis screening and evaluation

- Syphilis screening and evaluation

- HIV in pregnant women

- Pap smears

- Odynophagia/dysphagia

- Vision and HIV

- Fever clues

- Diarrhea clues

- Evaluation and treatment of diarrhea

- Evaluation and treatment of cough

\section{JOURNAL OF GENERAL INTERNAL MEDICINE SUBSCRIBERS Do we have your new address?}

Send us your new address three months before it becomes effective, so we will have time to get it into our computer system and ensure that your copies of JGIM continue to arrive uninterrupted. Send your old mailing label, your new address with zip code, the effective date of your new address, and your current telephone number.

\author{
Nonmember subscribers notify: \\ Rochelle Belanger \\ Blackwell Science, Inc. \\ Commerce Place, 350 Main St. \\ Malden, MA 02148
}

SGIM members notify:

Katrese Phelps

Society of General Internal Medicine 2501 M Street, NW, Suite 575

Washington, DC 20037 